Few-Body Syst manuscript No.

(will be inserted by the editor)

A. G. Volosniev • J. R. Armstrong • D. V. Fedorov ·

A. S. Jensen • N. T. Zinner

\title{
Bound Chains of Tilted Dipoles in Layered Systems
}

April 24, 2022

\begin{abstract}
Ultracold polar molecules in multilayered systems have been experimentally realized very recently. While experiments study these systems almost exclusively through their chemical reactivity, the outlook for creating and manipulating exotic few- and many-body physics in dipolar systems is fascinating. Here we concentrate on few-body states in a multilayered setup. We exploit the geometry of the interlayer potential to calculate the two- and three-body chains with one molecule in each layer. The focus is on dipoles that are aligned at some angle with respect to the layer planes by means of an external eletric field. The binding energy and the spatial structure of the bound states are studied in several different ways using analytical approaches. The results are compared to stochastic variational calculations and very good agreement is found. We conclude that approximations based on harmonic oscillator potentials are accurate even for tilted dipoles when the geometry of the potential landscape is taken into account.
\end{abstract}

\section{Introduction}

The prospect of extending the potential of ultracold quantum gases as a quantum simulation device into the realm of long-range interacting systems has received a massive boost with the recent success in producing cold polar molecules in their rotational and vibrational ground state $[1,2,3,4,4,5,6,7,8,8,9$. Systems of this type hold great promise for exploration of non-trivial few- and many-body dynamics with both fermions and bosons [10,11]. Three-dimensional samples with dipoles can unfortunately be highly unstable to chemical reactions losses [7. Trapping the dipoles in low-dimensional geometries have therefore been proposed to counteract this problem [12,13,14,15, 16, and recent experiments at JILA find large geometric effects that confirm a reduced loss in quasi-2D layers [17 and in three-dimensional optical lattices 18. The layered geometry with dipolar molecules has attracted much attention and exotic many-body phases such as paired states $[19,20,21,22,23,24,25$, interlayer coherence akin to ferromagnetism [26], anisotropic superfluidity [27], density waves [28, 29, 30, 31,32, 33, and non-trivial Fermi liquids 34,35, 36. However, the few-body structure is equally intriguing and bound complexes have been found with dipoles perpendicular to the plane in bi- [37, 38, 39, 40, 41] and multilayers [45, 46], for tilted dipoles in single 42 and bilayer setups [43,44, and in one-dimensional tubular geometries 47, 48, 49, 50, 51.

In this paper we focus on a few-body problem in a multilayered stack of planes when the dipoles are not perpendicular to the layer planes. Here we focus on the case of three adjacent planes, but we will comment on the implications for more layeres at the end of the presentation. A schematic view of the geometry and the dipoles is shown in Fig. 1. The energy and spatial structure of these two- and three-body dipolar complexes is our interest and we will use analytical approximations to the potentials to construct a harmonic model that is exactly solvable. However, this requires careful consideration of

Department of Physics and Astronomy, Aarhus University, DK-8000 Aarhus C, Denmark 
the geometry of the potential landscape of the molecules. To check how well an exact harmonic model can describe the system, we performed full stochastic variational calculations in a novel manner that takes the deformation of the potential for non-perpendicular dipoles into account. Our findings clearly demonstrate that the harmonic approach is very accurate for intermediate and strong dipole strength when the potential geometry is included properly. This holds for most tilting angles and only breaks down when the dipoles are oriented parallel to the layer. Our finding imply that away from the weakly interacting limit, the harmonic approximation works well in layered dipolar systems also in the case where the dipoles are tilted. This can be used in exact $N$-body harmonic Hamiltonian studies [52, 53. of both energetics, thermodynamics, and instabilities in larger systems [13, 45,54, with more layers which is the case for recent experiments with around 30 layers [17.

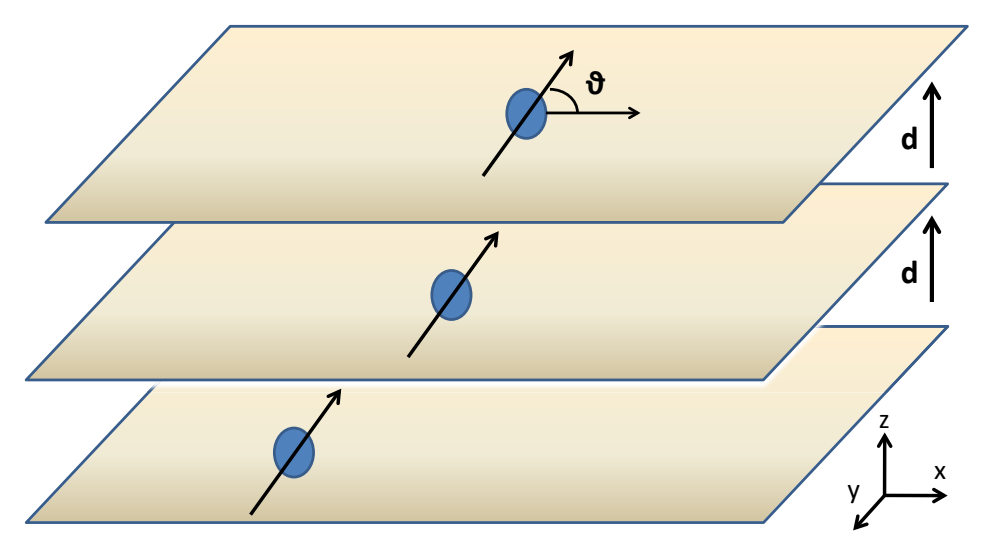

Fig. 1 Schematic picture of the trilayer geometry. The layer distance is $d$ and the coordinates we use are indicated in the bottom right-hand corner. The angle between the layer planes and the externally aligned dipole moment is $\theta$ and is taken to lie in the $x z$-plane.

\section{Model and results}

The dipolar potential between two dipoles with dipole moment $D$ in adjacent layers seperated by a distance $d$ is

$$
V(x, y)=D^{2} \frac{x^{2}+y^{2}+d^{2}-3(x \cos (\theta)+d \sin (\theta))^{2}}{\left(x^{2}+y^{2}+d^{2}\right)^{5 / 2}},
$$

where $\theta$ is the angle between the dipole direction and the layer plane as shown in Fig. 1. Below we will use $d$ as the unit for length, and $U=m D^{2} / \hbar^{2} d$ as the dipolar strength with $m$ the mass of the molecules. Energies will then be in units of $\hbar^{2} / m d^{2}$. The molecules used in recent experiments [17] are $\mathrm{KRb}$ which has a maximum dipole of 0.566 Debye. With the setup in Ref. [17, this gives a maximum $U \sim 1.2$ which is in the weak-coupling limit. However, molecules like LiCs or RbCs which are currently being cooled have much larger dipole moments 55 and increasing $U$ by factor of 10 or more should be possible with those species. Here we consider the strict two-dimensional limit where we can neglect the width of the layers. This holds when the optical lattice potential producing the layers is deep. Corrections to this can easily be taken into account by integrating out the transverse ( $z$-direction) degree of freedom with the wave function of the lattice (simply a Gaussian when the lattice is deep).

For $\theta=\pi / 2$ the potential is spherically symmetric, but for all other angles there is a distinct geometric structure. In Fig. 2 we show a contour plot of the potential for $\theta=\pi / 4$ where a clear 
asymmetric structure in the $x$-direction is visible. It is this fact that we can exploit to build an accurate analytical approximation to the ground-state wave function of the two- and three-body systems. Notice that there will be a three-body bound state for any value of $U$ in this setup with one molecule in each of the three layers. This can be understood from the fact that the two-body bound state in the case of two layers always exists as discussed in Refs. 44,43. Adding the third layer and molecule will then render the binding energy even smaller, i.e. $E_{3}<E_{2}$. This is a simple generalization of the proof given in Ref. [56] to the layered dipolar case where $\int d x d y V(x, y)=0$.

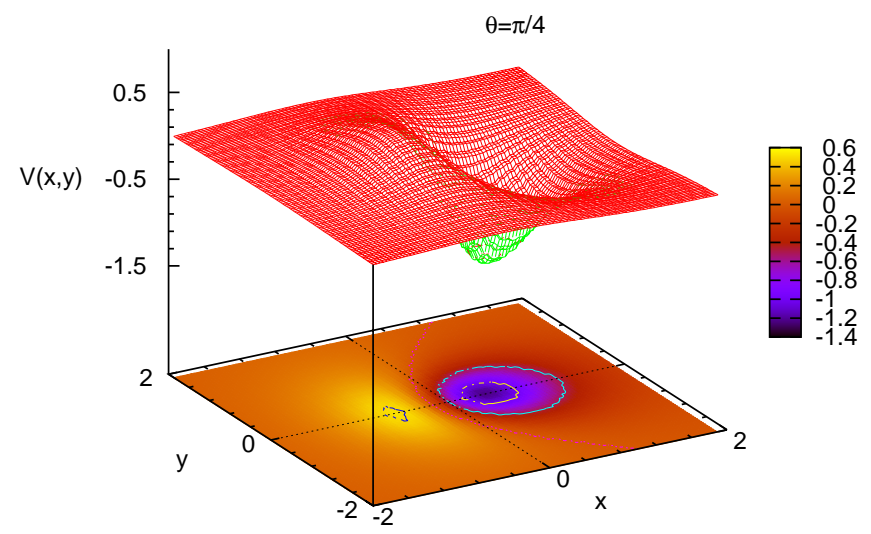

Fig. 2 Contour plot of the interlayer potential for $\theta=\pi / 4$ and $U=1$. The coordinates $x$ and $y$ are measured in units of $d$. Notice the strong asymmetry of the potential landscape in the $x$-direction. The potential has reflection symmetry along the $y$-direction.

To further illustrate the interesting geometrical structure of the potential along the $x$-direction, Fig. 3 displays the potential for $y=0$ and various values of $\theta$ between zero and $\pi / 2$. For $\theta=0$, there are two global minima located on each side of $x=0$, whereas for $\theta=\pi / 2$ we have a single minimum at $x=0$ and also cylindrical symmetry. In the regime $0<\theta \leq \theta_{c}^{*} \sim 0.955\left(\cos ^{2} \theta_{c}^{*}=\frac{1}{3}\right)$, the potential has two minima, one is located at $x>0$ and is deep, while the other one has a small minimum for $x<0$. At the intermediate angle $\theta_{c}\left(\sin ^{2} \theta_{c}=\frac{1}{3}\right)$ the potential is maximally asymmetric since here the monopole term vanishes 43. The monopole term can be deduced by expanding the potential in a two-dimensional spherical expansion via terms of the form $\cos \phi$ where $x=r \cos \phi$ and $y=r \sin \phi$ with $r=\sqrt{x^{2}+y^{2}}$ (the $y \leftrightarrow-y$ symmetry of the potential excludes $\sin \phi$ terms). The potential can be expanded using the basis function $1, \cos \phi$, and $\cos 2 \phi$ only, these correspond to a monopole, dipole, and quadrupole term as discussed in detail in Ref. 43 .

We want to exploit the geometry of the interlayer potential to determine the energy and structure of the bound states. This can be done by considering expansions of the Hamiltonian that use the minima of the potential (on the $x>0$ side) as the starting point. Denote the minimum position by $(x, y)=(a, 0)$, where $a$ is defined through the condition $\frac{\partial V(x, 0)}{\partial x}=0$. The equation determining $a$ is

$$
15 a(a \cos (\theta)+d \sin (\theta))^{2}-3 a\left(a^{2}+d^{2}\right)-6 \cos (\theta)\left(a^{2}+d^{2}\right)(a \cos (\theta)+d \sin (\theta))=0,
$$

from which one can easily see that $a$ scales as $d$, e.g. $a=d a_{0}$, where $a_{0}$ is the solution of Eq. (2) for $d=1$.

The potential can now be written in term of the variable $w=x-a$ centered at the minimum

$$
V(w, y)=D^{2} \frac{w^{2}+y^{2}+d^{2}+a^{2}+2 w a-3(w \cos (\theta)+d \sin (\theta)+a \cos (\theta))^{2}}{\left(w^{2}+y^{2}+d^{2}+a^{2}+2 w a\right)^{5 / 2}} .
$$

If we expand this to second order in $w$ and $y$ we have

$$
V(w, y)=v(d)+\alpha(d) w^{2}+\beta(d) y^{2} .
$$




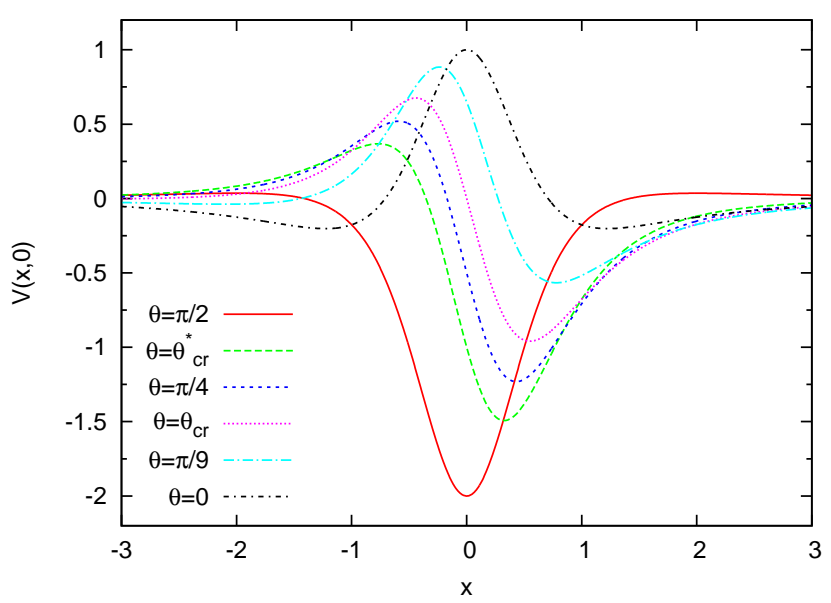

Fig. 3 Interlayer potential for $y=0$ and for different values of $\theta$ for $U=1$. The two special angles are defined through the relations $\cos ^{2} \theta_{c}^{*}=\frac{1}{3}$ and $\sin ^{2} \theta_{c}=\frac{1}{3}$.

where

$$
\begin{gathered}
v(d)=\frac{D^{2}}{d^{3}} v_{0}, v_{0}=\frac{a_{0}^{2}+1-3\left(\sin (\theta)+a_{0} \cos (\theta)\right)^{2}}{\left(a_{0}^{2}+1\right)^{5 / 2}}, \\
\alpha(d)=\frac{D^{2}}{d^{5}} \alpha_{0}, \\
\alpha_{0}=\frac{1-3 \cos ^{2}(\theta)+\frac{\left(30 a_{0}^{2}-5\right)\left(1+a_{0}^{2}-3\left(\sin (\theta)+a_{0} \cos (\theta)\right)^{2}\right)}{2\left(a_{0}^{2}+1\right)}-5 \frac{a_{0}\left(2 a_{0}-6 \cos (\theta)\left(a_{0} \cos (\theta)+\sin (\theta)\right)\right)}{2\left(a_{0}^{2}+1\right)^{2}}}{\left(a_{0}^{2}+1\right)^{5 / 2}}, \\
\beta(d)=\frac{D^{2}}{d^{5}} \beta_{0}, \beta_{0}=-\frac{3}{2} \frac{1+a_{0}^{2}-5\left(a_{0} \cos (\theta)+\sin (\theta)\right)^{2}}{\left(a_{0}^{2}+1\right)^{7 / 2}}
\end{gathered}
$$

Here we have used the scaling property $a=d a_{0}$ to extract the dependence on $d$ in each term. This will be convenient below.

\subsection{Harmonic three-body chains}

Consider three adjacent layers with interlayer distance $d$ as shown in Fig. 1. The potential for a threeparticle chain system with one particle in each layer using the harmonic approximation above is

$$
\begin{gathered}
V\left(x_{1}, x_{2}, x_{3}, y_{1}, y_{2}, y_{3}\right)=\frac{17 D^{2} v_{0}}{8 d^{3}}+\frac{D^{2} \alpha_{0}}{d^{5}}\left(x_{1}-x_{2}-d a_{0}\right)^{2}+\frac{D^{2} \beta_{0}}{d^{5}}\left(y_{1}-y_{2}\right)^{2} \\
+\frac{D^{2} \alpha_{0}}{d^{5}}\left(x_{2}-x_{3}-d a_{0}\right)^{2}+\frac{D^{2} \beta_{0}}{d^{5}}\left(y_{2}-y_{3}\right)^{2}+\frac{D^{2} \alpha_{0}}{(2 d)^{5}}\left(x_{1}-x_{3}-2 d a_{0}\right)^{2}+\frac{D^{2} \beta_{0}}{(2 d)^{5}}\left(y_{2}-y_{3}\right)^{2} .
\end{gathered}
$$

Notice the subtraction by $2 a_{0}$ in the term relating the $x_{1}$ and $x_{3}$ coordinates. This reflects the expected geometrical structure of the bound chain. Focus now on the $x$-dependent terms. Introducing the relative coordinate set $x_{1}-x_{3}=\sqrt{2} q_{1 x}, x_{1}-x_{2}=\sqrt{\frac{1}{2}} q_{1 x}+\sqrt{\frac{3}{2}} q_{2 x}$, and $x_{3}-x_{2}=-\sqrt{\frac{1}{2}} q_{1 x}+\sqrt{\frac{3}{2}} q_{x 2}$, we have

$$
\begin{gathered}
V_{x}\left(q_{1 x}, q_{2 x}\right)=\frac{D^{2} \alpha_{0}}{d^{5}}\left(\sqrt{\frac{1}{2}} q_{1 x}+\sqrt{\frac{3}{2}} q_{2 x}-d a_{0}\right)^{2}+\frac{D^{2} \alpha_{0}}{d^{5}}\left(-\sqrt{\frac{1}{2}} q_{1 x}+\sqrt{\frac{3}{2}} q_{2 x}+d a_{0}\right)^{2} \\
+\frac{D^{2} \alpha_{0}}{(2 d)^{5}}\left(\sqrt{2} q_{1}-2 d a_{0}\right)^{2}=\frac{17}{8} \frac{D^{2} \alpha_{0}}{d^{5}}\left(d a_{0}\right)^{2}+\frac{17}{16} \frac{D^{2} \alpha_{0}}{d^{5}} q_{1 x}^{2}+3 \frac{D^{2} \alpha_{0}}{d^{5}} q_{2 x}^{2}-\frac{17}{8} \frac{D^{2} \alpha_{0}}{d^{5}} \sqrt{2} q_{1 x} d a_{0} \\
=\frac{17}{16} \frac{D^{2} \alpha_{0}}{d^{5}}\left(q_{1 x}-\sqrt{2} d a_{0}\right)^{2}+3 \frac{D^{2} \alpha_{0}}{d^{5}} q_{2 x}^{2} .
\end{gathered}
$$

The ground-state energy in this oscillator potential is simply $E_{x}=\frac{\hbar^{2}}{m d^{2}} \sqrt{U \alpha_{0}}\left(\sqrt{\frac{3}{2}}+\sqrt{\frac{17}{32}}\right)$ with one term from each of the one-dimensional oscillator coordinates $q_{1 x}$ and $q_{2 x}$. The $y$-direction will give 


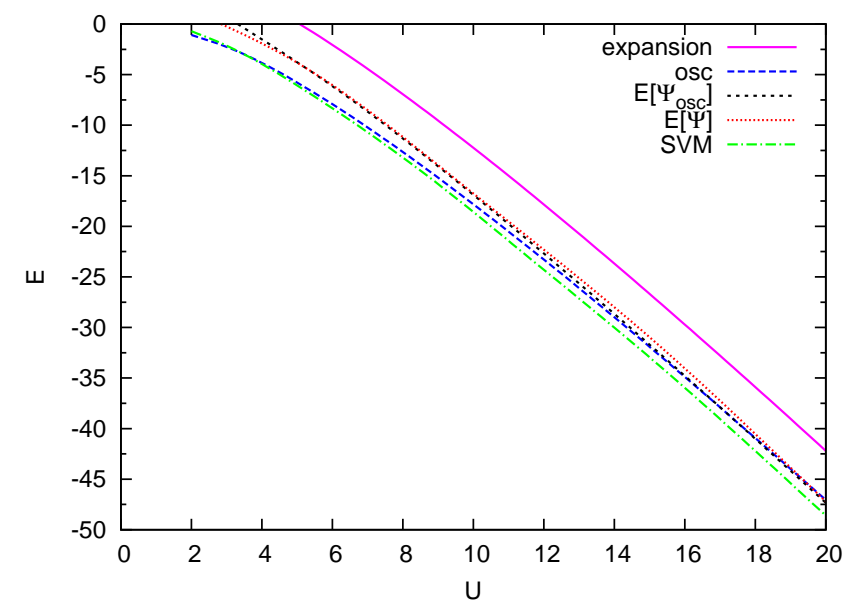

Fig. 4 Bound state energy of the three-body chain as function of dipolar strength $U$ for $\theta=\frac{\pi}{2}$. The dot-dashed line (SVM) is the value obtained from a numerical stochastic variational calculation. The full line (expansion) shows the result of Eq. $[10$, while the dotted $(E[\Psi])$ shows the result of using the wave function of Eq. (11) as a variational guess for the real dipolar potential of Eq. (1). The long-dashed (osc) is the optimized oscillator based on Eq. (14), while the short-dashed $\left(E\left[\Psi_{\mathrm{osc}}\right]\right)$ uses the wave function in Eq. 13 as a variational guess.

an identical contribution with $\beta_{0}$ instead of $\alpha_{0}$ and the final result for the ground-state energy of the three-chain, $E$, within this harmonic approximation is

$$
\frac{m d^{2}}{\hbar^{2}} E=\left(\sqrt{\frac{3}{2}}+\sqrt{\frac{17}{32}}\right)\left(\sqrt{\alpha_{0}}+\sqrt{\beta_{0}}\right) \sqrt{U}+\frac{17}{8} v_{0} U
$$

Below we will refer to this result for the energy as 'expansion' since it corresponds to a naive expansion of the potential around its minimum position to second order. The energies obtained from Eq. (10) are shown in Figs. 4 and 5 . For $\theta=\theta_{c}^{*}$, we find $a_{0}=\frac{3 \sqrt{17}-5}{2^{9 / 2}}$ which gives $\alpha_{0} \sim 2.66, \beta_{0} \sim 4.11$, and $v_{0} \sim-1.47$. In the perpendicular case where $\theta=\pi / 2$, we have $a_{0}=0, \alpha_{0}=\beta_{0}=6$, and $v_{0}=-2$.

The corresponding wave function in the 'expansion' method can be written

$$
\begin{aligned}
\Psi\left(q_{1 x}, q_{2 x}, q_{1 y}, q_{2 y}\right) & =N \exp \left[-\frac{1}{2} \sqrt{6 U \alpha_{0}}\left(\frac{q_{2 x}}{d}\right)^{2}-\frac{1}{2} \sqrt{\frac{17 U \alpha_{0}}{8}}\left(\frac{q_{1 x}}{d}-\sqrt{2} a_{0}\right)^{2}\right. \\
& \left.-\frac{1}{2} \sqrt{6 U \beta_{0}}\left(\frac{q_{2 y}}{d}\right)^{2}-\frac{1}{2} \sqrt{\frac{17 U \beta_{0}}{8}}\left(\frac{q_{1 y}}{d}\right)^{2}\right],
\end{aligned}
$$

where the normalization constant is $N^{2}=\sqrt{\frac{51}{4}} \sqrt{\alpha_{0} \beta_{0}} U / \pi^{2}$. This can be used to improve the approximation by considering instead the expectation value of the full potential from Eq. (1) and calculating $E[\Psi]=\frac{\langle\Psi|H| \Psi\rangle}{\langle\Psi \mid \Psi\rangle}$. The result of this proceduce is also shown on Figs. 4 and 5 . In comparison to the result from Eq. [10], $E[\Psi]$ is closer to the full numerical result based on the stochastic variational method (SVM) 43]. This implies that a harmonic oscillator wave function is a good variational guess. The difference between Eq. $[10)$ and $E[\Psi]$ arises mainly because the kinetic energy is overestimated in the naive expansion.

To investigate further the performance of harmonic approximations to the dipolar potential, we now consider an optimization on the two-body interlayer potential where the minimum position $a$ is a variational parameter. We consider the energy functional

$$
E[G]=\frac{<G|H| G>}{<G \mid G>}
$$

where $H$ is the full Hamiltonian with the real potential from Eq. (1) and

$$
G=\exp \left(-\frac{\tilde{\alpha}}{2}\left(\frac{x_{1}-x_{2}-\tilde{a}}{d}\right)^{2}-\frac{\tilde{\beta}}{2}\left(\frac{y_{1}-y_{2}}{d}\right)^{2}\right)
$$




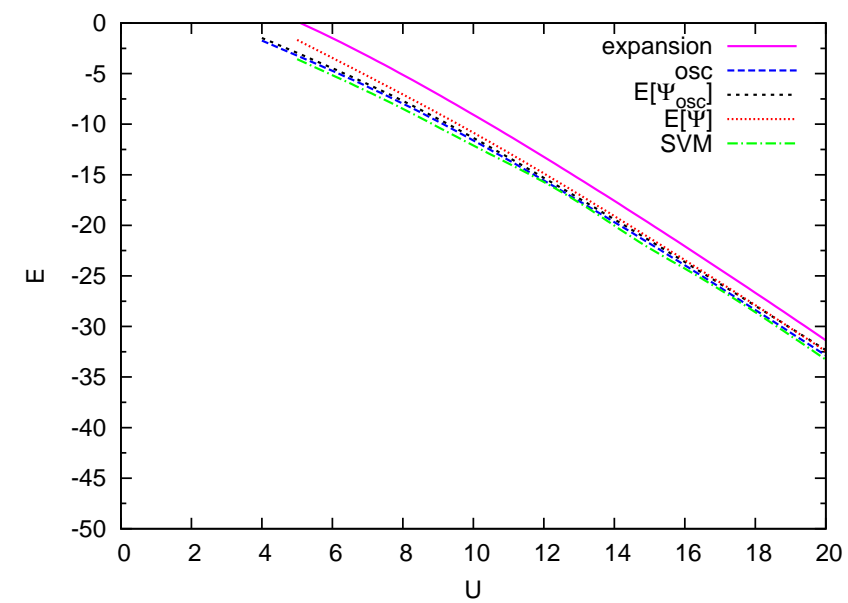

Fig. 5 Same as Fig. 4 but for $\theta=\theta_{c}^{*}$.

is a two-body wavefunction. Fixing $U$ and $\theta$, we can now minimize $E[G]$ to find the optimal set of $\tilde{\alpha}$, $\tilde{\beta}$, and $\tilde{a}$. This suggests what the optimal oscillator potential should be by replacing the real dipolar potential by a harmonic one of the form

$$
V_{\text {osc }}=\frac{\hbar^{2}}{2 m d^{2}} \tilde{\alpha}^{2}\left(\frac{x_{1}-x_{2}-\tilde{a}}{d}\right)^{2}+\frac{\hbar^{2}}{2 m d^{2}} \tilde{\beta}^{2}\left(\frac{y_{1}-y_{2}}{d}\right)^{2},
$$

which will give the energy $E_{\mathrm{osc}}=\frac{1}{2} \frac{\hbar^{2}}{m d^{2}}(\tilde{\alpha}+\tilde{\beta})$. This optimized harmonic potential for two particles in two adjacent layers can now be used as the starting point for the three-body calculations by replacing $\alpha_{0}, \beta_{0}$, and $a_{0}$ in Eq. (8) by $\tilde{\alpha}, \tilde{\beta}$, and $\tilde{a}$ (and dropping the constant term $v_{0}$ ). The results of this calculation is shown on Figs. 4 and 5 where it is labeled 'osc'. Just like above, one can use the three-body wave function obtained from this optimized choice of harmonic oscillator to calculate the expectation value of the real dipolar potential, the results of which is labeled ' $E\left[\Psi_{\mathrm{osc}}\right]$ '.

From Figs. 4 and Figs. 5 we see that our harmonic approximations are close (expect for the naive expansion) for $U \gtrsim 2(\theta=\pi / 2)$ and $U \gtrsim 4\left(\theta=\theta_{c}^{*}\right)$. Below these values, the wave function spread out too much to be described reliably by a single gaussian. Clearly, the naive expansion approach result from Eq. 10p performs the worst, although it appears to do somewhat better for tilted dipoles $(\theta<\pi / 2)$. However, using the wave function from Eq. 11) as a variational guess is a great improvement as demonstrated by the $E[\Psi]$ results. The optimzed oscillator results 'osc' and the corresponding variational guess based on this wave function, $E\left[\Psi_{\mathrm{osc}}\right]$, are both better than the expansion, and they differ only slightly. This is to be expected since the optimized oscillator will take as much of the real potential into account as possible using the three (essentially free) parameters $\tilde{\alpha}, \tilde{\beta}$, and $\tilde{a}$.

The fact that the result of Eq. $\sqrt{10}$ is rather poor, yet the wave function Eq. (11) is a good variational guess, is interesting. Note that the slope of the exact SVM and Eq. (10) is almost the same. This demonstrates that one can get a very accurate approximation for the energy by merely shifting the expansion result. This strategy has been pursued for $\theta=\pi / 2$ recently to study the properties of chains in multiple layers [45,54. The current results show that this approximation can be extended to the non-perpendicular case also.

To further investigate the structure we now look at the probability distribution for the three-body chain. The (partial) probability distribution for the first molecules is given by

$$
F\left(x_{1}, y_{1}\right)=\int\left|\Psi\left(q_{1 x}, q_{2 x}, q_{1 y}, q_{2 y}\right)\right|^{2} \delta\left(\frac{\mathbf{r}_{1}+\mathbf{r}_{2}+\mathbf{r}_{3}}{\sqrt{3}}\right) \mathrm{d} \mathbf{r}_{1} \mathrm{~d} \mathbf{r}_{2} \mathrm{~d} \mathbf{r}_{3},
$$

where we use the delta function to eliminate the integration over the coodinates of molecule $1, \mathbf{r}_{1}$, and similarly for the other molecules. In Fig. 6 we show the probability distributions in each layer for $\theta=\theta_{c}^{*}$ with $U=5$ and $U=15$ based on the gaussian wave functions used above (expansion method). This wave function very accurately describes the spatial structure of the full solution. The 

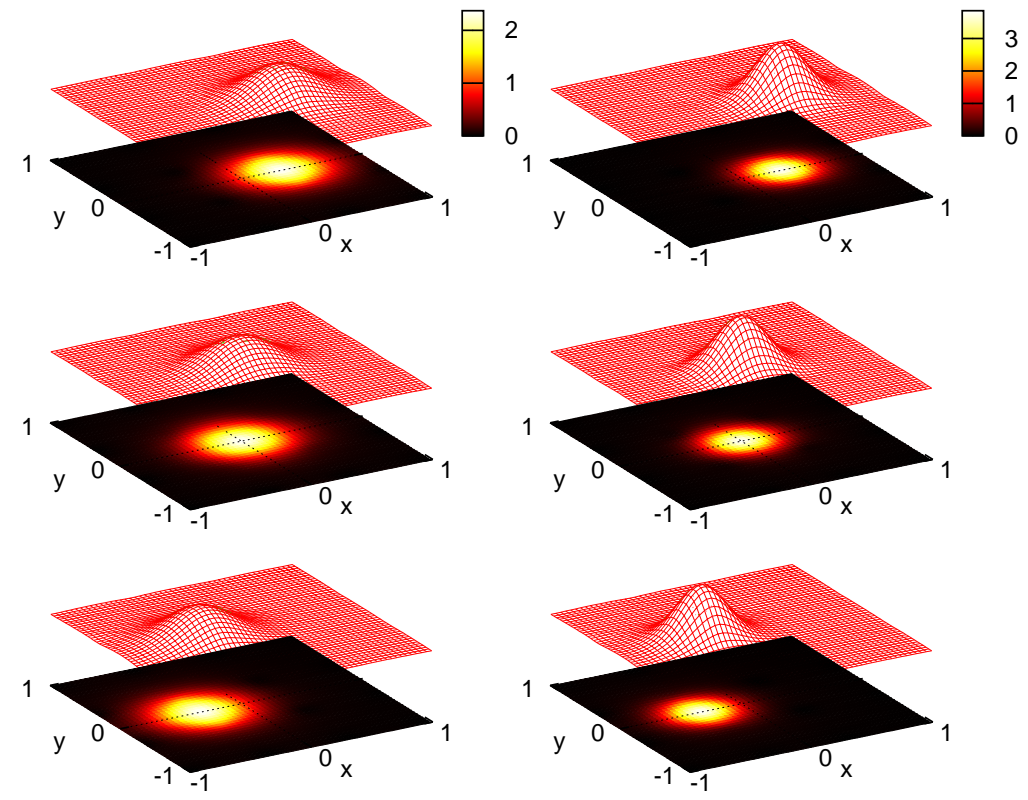

Fig. 6 Probability densities for the molecules in each of the layeres when $\theta=\theta_{c}^{*}$. Left side has $U=5$, while the right side has $U=15$.

figure clearly demonstrates the geometry of the dipolar potential with maximum in the middle of the center layer and displaced maxima in the two outer layers. This trend will continue for more than three layers. Notice also how the extend of the probability shrinks as $U$ increases in response to the increased localization in the potential minimum.

While we have only considered $\theta=\pi / 2$ and $\theta=\theta_{c}^{*}$ explicitly, similar results are obtained for a large range of angles $\theta>0$. Only close to $\theta=0$ do we run into problems since the potential has a two-minima structure which is not well described with a single gaussian for each molecule. Here one 
should presumably use at least two gaussians, one for each minimum. This is a subject for future investigations.

\section{Discussion}

We have studied polar molecules in a geometry consisting of equally spaced two-dimensional planes which is predicted to have a rich collection of few-body states due to the long-range and anisotropic dipole-dipole interaction between the molecules. In particular, the long-range character reaches across the different layers and provides an attractive force that can hold non-trivial bound states of several molecules. Here we have considered a simple case where there are three layeres and one molecule in each layer. This means that there are only interlayer interactions to worry about. When the dipole moment of the molecules are aligned perpendicular to the layers, a three-body bound chain is always present. While this result is expected to hold also when the dipoles are tilted away from perpendicular, no systematics has been considered thus far. Here we extend these results and show that for tilted dipoles the three-body chain persists and it can be well approximated by various gaussian wave function schemes. This conclusion can be easily extended to chains in setups with more than three layers.

Our results demonstrate that harmonic approximations to the dipolar interaction are very accurate in determining the bound state energy and also the spatial structure of the wave function in the intermediate and strong dipolar interaction regime also when the dipole moment is tilted with respect to the layers. Perhaps somewhat surprisingly, the harmonic approximation seems to work better around the critical angle (defined as the angle where two dipoles on a line will have zero interaction) than in the perpendicular case. We thus conclude that harmonic approximations are a viable and accurate strategy when studying the rich few-body structure of polar molecules in low-dimensional and/or lattice geometries.

\section{References}

1. Ospelkaus, S. et al.: Efficient state transfer in an ultracold dense gas of heteronuclear molecules. Nature Phys. 4, $622(2008)$

2. Ni, K.-K. et al.: A High Phase-Space-Density Gas of Polar Molecules. Science 322, 231 (2008)

3. Deiglmayr, J. et al.: Formation of Ultracold Polar Molecules in the Rovibrational Ground State. Phys. Rev. Lett. 101, 133004 (2008)

4. Lang, F., Winkler, K., Strauss, C., Grimm, R., Hecker Denschlag, J.: Ultracold Triplet Molecules in the Rovibrational Ground State. Phys. Rev. Lett. 101, 133005 (2008)

5. Carr, L.D., DeMille, D., Krems, R.V., Ye, J.: Cold and ultracold molecules: science, technology and applications. New J. Phys. 11, 055049 (2009)

6. Ni, K.-K. et al.: Dipolar collisions of polar molecules in the quantum regime. Nature 464 1324, (2010)

7. Ospelkaus, S. et al.: Quantum-State Controlled Chemical Reactions of Ultracold Potassium-Rubidium Molecules. Science 327 853, (2010)

8. Aikawa, K. et al.: Coherent Transfer of Photoassociated Molecules into the Rovibrational Ground State. Phys. Rev. Lett. 105, 203001 (2010)

9. Danzl, J. G. et al.: Quantum gas of rovibronic ground-state molecules in an optical lattice. Nature Phys. 6, 265 (2010)

10. Baranov, M. A.: Theoretical progress in many-body physics with ultracold dipolar gases. Phys. Rep. 464, $71(2008)$

11. Lahaye, T., Menotti, C., Santos, L., Lewenstein, M., Pfau, T.: The physics of dipolar bosonic quantum gases. Rep. Prog. Phys. 72, 126401 (2009)

12. Fischer, U.R.: Stability of quasi-two-dimensional Bose-Einstein condensates with dominant dipole-dipole interactions. Phys. Rev. A 73, 031602(R) (2006)

13. Wang, D.-W., Lukin, M.D., Demler, E.: Quantum Fluids of Self-Assembled Chains of Polar Molecules. Phys. Rev. Lett. 97, 180413 (2006)

14. Micheli, A., Pupillo, G., Büchler, H.P., Zoller, P.: Cold polar molecules in two-dimensional traps: Tailoring interactions with external fields for novel quantum phases. Phys. Rev. A 76, 043604 (2007)

15. Büchler, H.P. et al.: Strongly Correlated 2D Quantum Phases with Cold Polar Molecules: Controlling the Shape of the Interaction Potential. Phys. Rev. Lett. 98, 060404 (2007)

16. Gorshkov, A.V. et al.: Suppression of Inelastic Collisions Between Polar Molecules With a Repulsive Shield. Phys. Rev. Lett. 101, 073201 (2008)

17. de Miranda, M.H.G. et al.: Controlling the quantum stereodynamics of ultracold bimolecular reactions. Nature Phys. 7, 502 (2011)

18. Chotia, A. et al:: Long-lived dipolar molecules and Feshbach molecules in a 3D optical lattice. arXiv: $1110.4420 \mathrm{v} 1$ 
19. Bruun, G.M., Taylor, E.: Quantum Phases of a Two-Dimensional Dipolar Fermi Gas. Phys. Rev. Lett. 101, $245301(2008)$

20. Cooper, N.R., Shlyapnikov, G.V.: Stable Topological Superfluid Phase of Ultracold Polar Fermionic Molecules. Phys. Rev. Lett. 103, 155302 (2009)

21. Potter, A. C. et al:: Superfluidity and dimerization in a multilayered system of fermionic polar molecules. Phys. Rev. Lett. 105, 220406 (2010)

22. Pikovski, A.,Klawunn, M., Shlyapnikov, G.V., Santos, L.: Interlayer Superfluidity in Bilayer Systems of Fermionic Polar Molecules. Phys. Rev. Lett. 105, 215302 (2010)

23. Zinner, N.T., Wunsch, B., Pekker, D., Wang, D.W.: BCS-BEC Crossover in Bilayers of Cold Fermionic Polar Molecules. Phys. Rev. A. 85, 013603 (2012)

24. Baranov, M.A., Micheli, A., Ronen, S., Zoller, P.: Bilayer superfluidity of fermionic polar molecules: Manybody effects. Phys. Rev. A 83, 043602 (2011)

25. Levinsen, J., Cooper, N.R., Shlyapnikov, G.V.: Topological px+ipy superfluid phase of fermionic polar molecules. Phys. Rev. A 84, 013603 (2011)

26. Lutchyn, R.M., Rossi, E., Das Sarma, S.: Spontaneous interlayer superfluidity in bilayer systems of cold polar molecules. Phys. Rev. A 82, 061604(R) (2010)

27. Ticknor, C., Wilson, R.M., Bohn, J.L.: Anisotropic Superfluidity in a Dipolar Bose Gas. Phys. Rev. Lett. 106, $065301(2011)$

28. Sun, K., Wu, C., Das Sarma, S.: Spontaneous inhomogeneous phases in ultracold dipolar Fermi gases. Phys. Rev. B 82, 075105 (2010)

29. Yamaguchi, Y., Sogo, T., Ito, T., Miyakawa, T.: Density-wave instability in a two-dimensional dipolar Fermi gas. Phys. Rev. A 82, 013643 (2010)

30. Zinner, N.T., Bruun, G.M.:Density Waves in Layered Systems with Fermionic Polar Molecules. Eur. Phys. $\mathrm{J} \mathrm{D}$, in press.

31. Babadi, M., Demler, E.: Density ordering instabilities of quasi-two-dimensional fermionic polar molecules in single-layer and multi-layer configurations: exact treatment of exchange interactions. Phys. Rev. B 84, $235124(2011)$

32. Sieberer, L. M., Baranov, M. A.: Collective modes, stability and superfluid transition of a quasi-twodimensional dipolar Fermi gas. Phys. Rev. A 84, 063633 (2011)

33. Parish, M. M., Marchetti, F. M.: Density instabilities in a two-dimensional dipolar Fermi gas. arXiv: 1109.2464

34. Chan, C.K., Wu, C.J., Lee, W.C., Das Sarma, S.: Anisotropic-Fermi-liquid theory of ultracold fermionic polar molecules: Landau parameters and collective modes. Phys. Rev. A 81, 023602 (2010)

35. Kestner, J.P., Das Sarma, S.: Compressibility, zero sound, and effective mass of a fermionic dipolar gas at finite temperature. Phys. Rev. A 82, 033608 (2010)

36. Lu, Z.-K., Shlyapnikov, G. V.: Novel Fermi Liquid of 2D Polar Molecules. arXiv:1111.7114v1.

37. Shih, S.M., Wang, D.W.: Pseudopotential of an interaction with a power-law decay in two-dimensional systems. Phys. Rev. A 79, 065603 (2009)

38. Armstrong, J.R., Zinner, N.T., Fedorov, D.V., Jensen, A.S.: Bound states and universality in layers of cold polar molecules. Europhys. Lett. 91, 16001 (2010)

39. Klawunn, M., Pikovski, A., Santos, L.: Two-dimensional scattering and bound states of polar molecules in bilayers. Phys. Rev. A 82, 044701 (2010)

40. Fedorov, D.V., Armstrong, J.R., Zinner, N.T., Jensen, A.S.: Weakly bound states of polar molecules in bilayers. Few-body Syst. 50, 417 (2011)

41. Zinner, N.T., Armstrong, J.R., Volosniev, A.G., Fedorov, D.V., Jensen, A.S.: Dimers, Effective Interactions, and Pauli Blocking Effects in a Bilayer of Cold Fermionic Polar Molecules. arXiv:1105.6264

42. Cremon, J.C., Bruun, G.M., Reimann, S.M.: Tunable Wigner States with Dipolar Atoms and Molecules. Phys. Rev. Lett. 105, 255301 (2010)

43. Volosniev, A.G. et al.: Bound dimers in bilayers of cold polar molecules. J. Phys. B 44, 125301 (2011)

44. Volosniev, A.G., Fedorov, D.V., Jensen, A.S., Zinner, N.T.: Model Independence in Two Dimensions and Polarized Cold Dipolar Molecules. Phys. Rev. Lett. 106, 250401 (2011)

45. Armstrong, J.R., Zinner, N.T., Fedorov, D.V., Jensen, A.S.: Layers of Cold Dipolar Molecules in the Harmonic Approximation. arXiv:1106.2102 v1

46. Volosniev, A. G., Fedorov, D. V., Jensen, A. S., Zinner, N. T.: Few-body bound state stability of dipolar molecules in two dimensions. Phys. Rev. A 85, 023609 (2012)

47. Deuretzbacher, F., Cremon, J.C., Reimann, S.M.: Ground-state properties of few dipolar bosons in a quasi-one-dimensional harmonic trap. Phys. Rev. A 81, 063616 (2010)

48. Klawunn, M., Duhme, J., Santos, L.: Bose-Fermi mixtures of self-assembled filaments of fermionic polar molecules. Phys. Rev. A 81, 013604 (2010)

49. Wunsch, B., Zinner, N.T., Mekhov, I.B., Huang, S.J., Wang, D.W., Demler, E.: Few-body bound states in dipolar gases and their detection. Phys. Rev. Lett. 107, 073201 (2011)

50. Zinner, N.T., Wunsch, B., Mekhov, I.B., Huang, S.J., Wang, D.W., Demler, E.: Few-Body Bound Complexes in One-dimensional Dipolar Gases and Non-Destructive Optical Detection. Phys. Rev. A 84, 063606 (2011)

51. Mekhov, I.B.: Quantum Non-Demolition Detection of Polar Molecule Complexes: Dimers, Trimers, Tetramers. arXiv:1111.3908

52. Armstrong, J.R., Zinner, N.T., Fedorov, D.V., Jensen, A.S.: Analytic Harmonic Approach to the N-body problem. J. Phys. B: At. Mol. Opt. Phys. 44, 055303 (2011)

53. Armstrong, J.R., Zinner, N.T., Fedorov, D.V., Jensen, A.S.: Quantum statistics and thermodynamics in the harmonic approximation. Phys. Rev. E 85, 021117 (2012). 
54. Armstrong, J.R., Zinner, N.T., Fedorov, D.V., Jensen, A.S.: Thermodynamics of Dipolar Chain Systems. arXiv:1112.6141

55. Aymar, A., Dulieu, O.: Calculation of accurate permanent dipole moments of the lowest ${ }^{1,3} \Sigma^{+}$states of heteronuclear alkali dimers using extended basis sets. J. Chem. Phys. 122, 204302 (2005)

56. Coutinho, F.A.B., Malta, C.P., Fernando Perez, J.: On the existence of bound states of $N$-particle systems in one and two dimensions. Phys. Lett. A 97, 242 (1983) 